\title{
Effect of Electro-acupuncture on Vasomotor Symptoms in Rats with Acute Cerebral Infarction Based on Phosphatidylinositol System*
}

\author{
LI Jing ${ }^{1}$, HE Ying1', DU Yuan-hao², ZHANG Min ${ }^{1}$, Rainer Georgi ${ }^{3}$, Bernhard Kolberg ${ }^{4}$, \\ SUN Dong-wei ${ }^{5}$, MA Kun ${ }^{6}$, LI Yong-feng ${ }^{7}$, and ZHANG Xue-zhu ${ }^{1}$
}

\begin{abstract}
Objective: To investigate the effect of electro-acupuncture (EA) on vasomotor symptoms in rats with acute cerebral infarction, by observing the changes in the expression of factors related to the phosphatidylinositol (PI) system. Methods: Forty-two Wistar rats were randomly divided into 3 groups by a random number table: the control group $(n=6)$, the model group $(n=18)$ and the EA group $(n=18)$. The EA group was given EA treatment at Shuigou (GV 26) instantly after modeling with middle cerebral artery occlusion (MCAO) method, while the model and control groups were not given any treatment. The degrees of neurological deficiency were evaluated using neurological severity scores (NSS) and the brain blood flow was evaluated by a laser scanning confocal microscope. Western blot analysis was conducted to detect the expression levels of G-protein subtype (Gq) and calmodulin (CaM). Competition for protein binding was conducted to detect the expression level of inositol triphosphate (IP3). Thin layer quantitative analysis was conducted to detect the expression level of diacylglycerol (DAG). The expression level of intracellular concentration of free calcium ion $\left(\left[\mathrm{Ca}^{2+}\right]\right.$ ) was detected by flow cytometry. Results: The NSS of the model group was significantly higher than the control group at 3 and $6 \mathrm{~h}$ after MCAO $(P<0.01)$, while the EA group was significantly lower than the model group at $6 \mathrm{~h}(P<0.01)$. The cerebral blood flow in the model group was significantly lower than the control group at 1,3 and $6 \mathrm{~h}$ after MCAO $(P<0.01)$, while for the EA group it was remarkably higher than the model group at the same time points $(P<0.01)$. The expressions of Gq, CaM, IP3, DAG and $\left[\mathrm{Ca}^{2+}\right]_{i}$ in the model group were significantly higher than the control group $(P<0.05$ or $P<0.01)$, and those in the EA group were significantly lower than the model
\end{abstract} group at the same time points $(P<0.05$ or $P<0.01)$. Conclusion: EA treatment at GV 26 can effectively decrease the over-expression of related factors of PI system in rats with acute cerebral infarction, improve cerebral autonomy movement, and alleviate cerebral vascular spasm.

KEYWORDS cerebral infarction, phosphatidylinositol system, vasomotion, Shuigou (GV 26), electroacupuncture, Chinese medicine

Cerebral infarction is a disease with a complex pathophysiology. The infarcted brain often results from focal cerebral ischemia. ${ }^{(1)}$ The cerebral blood flow accounts for $13 \%$ of the cardiac blood flow output, which combined with the fact that the brain tissue cannot store blood flow, making it crucial for the brain to get the compensatory blood flow in a timely and effective manner. It can decide the fate of the brain cell after the onset of cerebral vascular occlusion. ${ }^{(2)}$ The number of microvessels in the infarcted brain tissue plays an important role in the establishment of

(C)The Chinese Journal of Integrated Traditional and Western Medicine Press and Springer-Verlag GmbH Germany, part of Springer Nature 2021

*Supported by the National Natural Science Foundation of China (Nos. 82074543, 81473765, 81674056) and the Tianjin Municipal Natural Science Foundation (No. 18JCYBJC94200)

1. Key Laboratory of Acupuncture of Tianjin, First Teaching Hospital of Tianjin University of Traditional Chinese Medicine, National Clinical Research Center for Chinese Medicine Acupuncture and Moxibustion, Tianjin (300381), China; 2. Department of Acupuncture and Moxibustion, First Teaching Hospital of Tianjin University of Traditional Chinese Medicine, Tianjin (300381), China; 3. Department of Chemical Biology, Max Planck Institute for Medical Research, Heidelberg (69120), Germany; 4. Department of Internal Medicine, Mannheim Medical School of Heidelberg University, Mannheim (68167), Germany; 5. Department of Chinese Medicine Rehabilitation, Shenzhen Baoan District Hospital of Traditional Chinese Medicine, Shenzhen, Guangdong Province (518000), China; 6. Department of Preventive Treatment of Disease, Binhai New Area Hospital of Traditional Chinese Medicine, Fourth Teaching Hospital of Tianjin University of Traditional Chinese Medicine, Tianjin (300193), China; 7. Institute of Acupuncture and Moxibustion, Shaanxi University of Chinese Medicine, Xi'an (712046), China

Correspondence to: Prof. DU Yuan-hao, E-mail: ddyh64 @ tjutcm.edu.cn

DOI: https://doi.org/10.1007/s11655-021-3341-6 
collateral circulation in the cerebrovascular system. ${ }^{(3)}$ The phosphatidylinositol (PI) system is responsible for signal transmission and regulation of vasomotor symptoms in the intracellular cerebral vasculature. ${ }^{(4)}$ Therefore, it is important to identify the factors as well as ways to modulate the microcirculation of the brain. Our previous studies have proved that the function and morphology of cerebral blood vessels were severely impaired after cerebral infarction, and stimulating the acupoint Shuigou (GV 26) could relax the cerebral blood vessels. ${ }^{(5-7)}$ This could then promote the collateral circulation on the surface of the brain, improve the autonomic movement of vasomotor of brain, increase the compensatory blood flow, and improve microcirculation of the brain. This research focuses on investigating whether electro-acupuncture (EA) treatment is related to the signal transduction pathway of $\mathrm{PI}$ system.

\section{METHODS}

Animals

Forty-two healthy Wistar male rats (weighing 180-200 g, 5-6 weeks old, specific-pathogenfree grade) were provided by Beijing Spaefer Biotechnology Co., Ltd., China [License No. SCXK (J) 2018/012]. Rats were raised at a controlled room temperature of $25{ }^{\circ} \mathrm{C}$ and relative humidity of $40 \%-70 \%$, with an alternating $12 \mathrm{~h} / 12 \mathrm{~h}$ light/dark cycle, at the Institute of Radiation Medicine, Chinese Academy of Medical Sciences, with adequate food and water, and avoid from being irradiated by strong light. This experiment was approved by the Animal Ethics Committee, Tianjin University of Traditional Chinese Medicine, China (approval No. TCMLAEC2019021), and was performed in accordance with the National Institutes of Health Guide for the Care and Use of Laboratory Animals.

\section{Grouping and Modeling}

Totally 42 rats were randomly divided into 3 groups by a random number table: control group $(n=6)$, model group ( $n=18)$ and EA group ( $n=18)$. The model and EA groups were further divided into 3 subgroups according to the time after middle cerebral artery occlusion (MCAO), i.e. 1,3 , and $6 \mathrm{~h}$, with 6 rats in each subgroup.

To replicate cerebral infarction model, MCAO was performed according to the method described by Longa, et al. ${ }^{(8)}$ Anesthetic masks were put on rats and the rats were anesthetized with isoflurane (induced anesthesia at $4 \%$, maintained at $2 \%$ ), and fixed on the operating table in supine position. Through a midline incision, the left common carotid artery (CCA), external carotid artery (ECA), and internal carotid artery (ICA) were separated, and the ECA was isolated and coagulated. Two microsurgical clips were placed at the proximal and furcal parts of the CCA, then nylon suture with a diameter of $0.205 \mathrm{~mm}$ and length of 18-22 $\mathrm{mm}$ was introduced into the lumen through a puncture that was pricked between two clips. After the clips were removed, the nylon suture was gently advanced into the ICA until resistance was felt. Then the incision was closed after the suture was tightened and the clips were loosened.

\section{Intervention}

Rats in the EA group were given EA treatment at GV 26 instantly after MCAO. GV 26 acupoint is located according to the "Development of Acupoint Atlas of Rats" described by Hua, et al. ${ }^{(9)}$ The first acupuncture needle ( $0.25 \mathrm{~mm} \times 13 \mathrm{~mm}$, Huatuo Brand, China) was inserted upward and obliquely $2 \mathrm{~mm}$ into the nasal septum, then the second needle was punctured into the location at $2 \mathrm{~mm}$ below GV 26 as the reference point. The needles inserted at GV 26 and the reference point were connected with the positive and negative electrode of the EA apparatus (HANS LH202H, Beijing Xingyu Hongye Trading Co., Ltd., China), respectively. The stimulus parameters of continuous-wave at $15 \mathrm{~Hz}$ and $1 \mathrm{~mA}$ were selected and continued for $20 \mathrm{~min}$. Rats in the model and control groups were not given any treatment.

\section{Neurological Severity Scores}

The neurological deficits of rats in each group were evaluated using neurological severity score (NSS, 0-18 points). ${ }^{(10)} \mathrm{NSS}$ is a comprehensive scoring index for motor, sensory, reflex and balance functions. The higher score corresponds to the severity of injury. Rats were scored for neurological deficits before sampling and those with NSS score $\geqslant 2$ points were considered as the neurological model. The dead rats and those with NSS score $<2$ points were excluded.

\section{Sampling and Laser Scanning Confocal Microscopy}

Rats were injected with fluorescently labeled (isothiocyanate fluorescein FITC, $50 \mathrm{mg} / \mathrm{mL}$ ) dextran from one side of the femur vein before sacrifice. After $1 \mathrm{~min}$, the rats were sacrificed by decapitation after anesthesia, brain tissues were removed instantly and 
fixed in $4 \%$ paraformaldehyde buffer solution overnight at $4{ }^{\circ} \mathrm{C}$. Coronal sections of $150 \mu \mathrm{m}$ thickness were cut from the interaural plane from $6.38 \mathrm{~mm}$ to $1.00 \mathrm{~mm}$ according to the rat brain map. Ten sections of each specimen were observed using MRC 1024 laser scanning confocal microscope system (BioRad, USA). The scanning conditions were as follows: excitation wavelength $488 \mathrm{~nm}$, emission wavelength $522 \mathrm{~nm}$, electron amplification to 2, ultraviolet excitation wavelength $361 \mathrm{~nm}$, emission wavelength 420-470 nm, laser intensity $463 \mathrm{~mW}$, scanning speed $10 \mathrm{~mm} / \mathrm{s}$, scanning step distance $0.3 \mu \mathrm{m}$, and sample points 9 . Each section was positioned and imaged according to the rat brain map, and the three-dimensional reconstruction was performed using the confocal system (Bio-Rad) to detect the brain blood flow.

\section{Western Blot Analysis}

Rats were anesthetized with isoflurane and decapitated immediately at the specific time points. Brain tissues were collected in phosphate buffered saline (PBS) and centrifuged at $4{ }^{\circ} \mathrm{C}(14,000 \times g, 5 \mathrm{~min})$. The total protein was quantified by bicinchoninic acid (BCA) method. ${ }^{(11)}$ Then $30 \mu \mathrm{L}$ of the processed protein sample was electrophoresed on a $10 \%$ sodium dodecyl sulfate-polyacrylamide gel electrophoresis (SDS-PAGE) gel and transferred to a polyvinylidene fluoride membrane. The standard protein markers were used as a reference, and the band was cut according to the molecular weight, and blocked with $5 \%$ skimmed milk. G-protein subtype $(\mathrm{Gq})$ antibody (Abcam, USA, 1:1000) and calmodulin (CaM) antibody (Abcam, 1:1000) were added to the respective bands, and incubated overnight at $4{ }^{\circ} \mathrm{C}$. After incubation with secondary antibody (Abcam, ab97023, ab6721) and the chemiluminescent reagent, the membrane was scanned with a scanner. The gray value of bands was analyzed using ImageJ 1.8 .0 software (National Institutes of Health, USA), and the ratio of the gray value of the target protein to the internal reference GAPDH and $\beta$-actin was calculated to represent the relative expression level of the target protein.

\section{Competitive Protein Binding Assay}

The main steps were as follows. ${ }^{(12)}$ The rats were sacrificed and brains tissues were removed instantly and fixed in ice. The Willis ring, anterior, middle and posterior cerebral arteries and their branches were taken out quickly with the application of microsurgical technique and stored at $-70{ }^{\circ} \mathrm{C}$. Then $150 \mathrm{mg}$ of cerebral arteries were cut $(2 \mathrm{~mm} \times 2 \mathrm{~mm})$, and incubated at $31{ }^{\circ} \mathrm{C}$ for $30 \mathrm{~min}$ in digestive solution. The digested cerebral arteries were centrifuged at $1,500 \mathrm{r} / \mathrm{min}, 20^{\circ} \mathrm{C}$ for $4 \mathrm{~min}$. The supernatant and the digested pellet were separated, and the pellet was subject to digestion again in the same conditions. The sample was passed through mesh sieves (200 mesh) to obtain free individual cells which were collected in $1.5 \mathrm{~mL}$ centrifuge tubes filled with $1 \mathrm{~L} / \mathrm{L}$ oxygen.

[3H]-inositol triphosphate (IP3) test kit (GSTIP3-bp, Amersham Biosciences Corp., UK) was used according to manufacturer's instructions. First, $500 \mu \mathrm{L}$ of cerebral arterial cells suspension was prepared in a centrifuge tube, to which $1 \mathrm{~mL}$ of trichloroacetic acid $(15 \%)$ was added at $4{ }^{\circ} \mathrm{C}$, followed by sonication $(3 \times 20$ s bursts) while being incubated in an ice water bath for $5 \mathrm{~min}$. Second, cell lysates were centrifuged at $4,000 \mathrm{r} / \mathrm{min}$ for $40 \mathrm{~min}$ at $4{ }^{\circ} \mathrm{C}$, to precipitate protein. Third, $1.5 \mathrm{~mL}$ of the water-saturated diethylene ether was added to $0.5 \mathrm{~mL}$ of the supernatant, and stirred till thoroughly incorporated, followed by centrifugation at 2,000 r/min for $10 \mathrm{~min}$. Fourth, the upper ethyl ether layer was discarded and the samples were washed thrice. The upper layer containing ethyl ether was discarded and the remaining samples were stored at $-20{ }^{\circ} \mathrm{C}$ for coagulation. The specimens were treated using protein competition combined analysis to measure the reaction time of radioactivity, on average count. The percentage binding rate of IP3 in standard tube and sample tube was calculated and converted into the amount of triphosphate in every $10^{6}$ cells to get triphosphate unit as pmol/10 ${ }^{6}$.

\section{Thin Layer Quantitative Analysis}

The sample preparation method is the same as that of competition for protein binding, the following steps were done. ${ }^{(13)}$ First, $2 \mathrm{~mL}$ of chloroform: methanol $(1: 2, v / v)$ solution was added to $500 \mu \mathrm{L}$ of cerebral artery vascular cell suspension in a centrifuge tube at $4{ }^{\circ} \mathrm{C}$ under $3 \times 20 \mathrm{~s}$ ultrasonic pulverization, and incubated in ice water for $20 \mathrm{~min}$. Second, samples were then centrifuged $(2,000 \mathrm{r} / \mathrm{min}, 10 \mathrm{~min})$, the liquid was collected, subsided, which was then washed with chloroform: methanol, and the supernatant was again collected after centrifugation. Third, $0.5 \mathrm{~mL}$ of distilled water and $0.5 \mathrm{~mL}$ of chloroform was added and the previous steps were repeated at least 4 times. Fourth, the collected chloroform phase was dried by blowing nitrogen and then refrigerated for testing. 
Fifth, $100 \mu \mathrm{L}$ of chloroform was added to the sample, and $10 \mu \mathrm{L}$ of the sample was spotted on a silica gel GF254 thin layer chromatography (TLC) plate, along with $0.1,0.3,0.6$, and $0.9 \mu \mathrm{g}$ of 1,2-diacylglycerol (DAG) standards which were spotted on the same TLC plate for calibration. Sixth, TLC plate was developed in cyclohexane: isopropyl ether: acetic acid (65:35:2, v/v), stained with Coomassie Brilliant Blue G-250 (R21264$500 \mathrm{~mL}$, Shanghai Yuanye Bio-Technology Co., Ltd., China). After decolorization of $20 \%$ methanol in 2 steps, the DAG was quantitatively determined by CS-9301 thin layer scanner (CAMAG Co., Switzerland). The detection wavelength was $584 \mathrm{~nm}$.

\section{Flow Cytometry}

The sample preparation method is the same as that of competition for protein binding. The main steps were as follows. ${ }^{(14)}$ The removed arterial ring was quickly placed in a $1.5 \mathrm{~mL}$ centrifuge tube to which $1,000 \mu \mathrm{L}$ of pre-cooled D-hanks solution was added. The blood vessel was cut with ophthalmic scissors, allowed to stand for $1 \mathrm{~min}$, and the supernatant was discarded. The pellet was suspended in $1 \%$ type I collagenase $5 \mathrm{~mL}$ digestion solution (stored at $4{ }^{\circ} \mathrm{C}$ ) and incubated at $37{ }^{\circ} \mathrm{C}$ in a water bath ( $1 \mathrm{~mL}$ of collagenase with a concentration of $10 \%$ was taken out, dissolved at room temperature, and $9 \mathrm{~mL}$ of D-hanks solution kept at $4{ }^{\circ} \mathrm{C}$, was added to prepare a $1 \%$ digestive solution). The sample was slightly shaken and allowed to digest for $30 \mathrm{~min}$. Then, the sample was passed through a 200-mesh sieve and the resulting filtrate was collected and centrifuged at 1,000 r/min for $7 \mathrm{~min}$ at $4{ }^{\circ} \mathrm{C}$. The supernatant was discarded and the pellet was suspended in $200 \mu \mathrm{L}$ of pre-cooled D-hanks solution and mix thoroughly. Then $100 \mu \mathrm{L}$ each of the fraction was placed in two 1.5-mL centrifuge tubes. To the first tube, $20 \mu \mathrm{L}$ of flu-3/AM (concentration: $10 \mu \mathrm{g} / \mathrm{L}$ ) was added, while the tube served as negative control. Both the tubes were simultaneously incubated at $37{ }^{\circ} \mathrm{C}$ for $30 \mathrm{~min}$. After incubation, $500 \mu \mathrm{L}$ of pre-cooled D-hanks solution was added to each tube to terminate the reaction, the samples were immediately analyzed using a flow cytometer (EPICS ALTRA, Beckman Coulter, USA). The fluorescence intensity (\%) after stimulation was read at $506 \mathrm{~nm}$ to reflect the change in intracellular concentration of free calcium ion $\left(\left[\mathrm{Ca}^{2+}\right]_{\mathrm{i}}\right)$ in the cells.

\section{Statistical Analysis}

Data were processed with SPSS 11.5 software (International Business Machines Corporation, USA) and expressed as mean \pm standard deviation $(\bar{x} \pm s)$. The $\left[\mathrm{Ca}^{2+}\right]_{i}$ data were evaluated by $t$-test, and the remaining data were analyzed using one-way analysis of variance (ANOVA) followed by the least-significant difference or Dunnett T3 method for analysis between two groups. Statistical significance was attributed at a $P$ value of $<0.05$.

\section{RESULTS}

\section{EA Reduced NSS in Rats with Acute Cerebral Infarction}

Due to the effects of anesthesia, the MCAO rats were not fully conscious at $1 \mathrm{~h}$, therefore, 3- and 6-h MCAO rats were included for NSS observation. The NSS of the control group was 0 point. The NSS of the model group was significantly higher than the control group at 3 and $6 \mathrm{~h}(P<0.01)$. The NSS of the EA group was significantly lower than that of the model group at $6 \mathrm{~h}(P<0.01$, Figure 1).

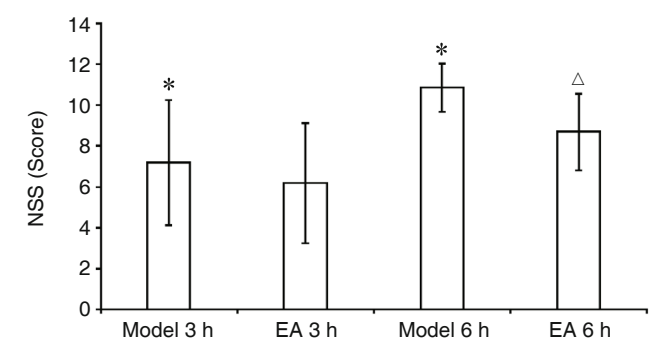

Figure 1. EA Reduced NSS in Rats with

Acute Cerebral Infarction $(\bar{x} \pm s, n=6)$

Notes: ${ }^{*} P<0.01$ vs. control group at the same time point; ${ }^{\Delta} P<0.01$ vs. model group at the same time point

\section{EA Induced Increase of Cerebral Blood Flow in} Rats with Acute Cerebral Infarction

The cerebral blood flow of the model group was significantly lower than the control group at different time points $(P<0.01)$, while the EA group was significantly higher than that of the model group at the same time points $(P<0.01$, Figure 2$)$.

\section{EA Decreased Protein Expressions of Gq and CaM in Rats with Acute Cerebral Infarction \\ The protein expressions of $\mathrm{Gq}$ and CaM in the model group were significantly higher than the control group at the same time points $(P<0.05)$, while the $E A$ group were significantly lower than the model group at the same time points $(P<0.05$, Figure 3$)$.}

\section{EA Reduced Contents of IP3 and DAG in Rats with Acute Cerebral Infarction}

The contents of IP3 and DAG in the model group 

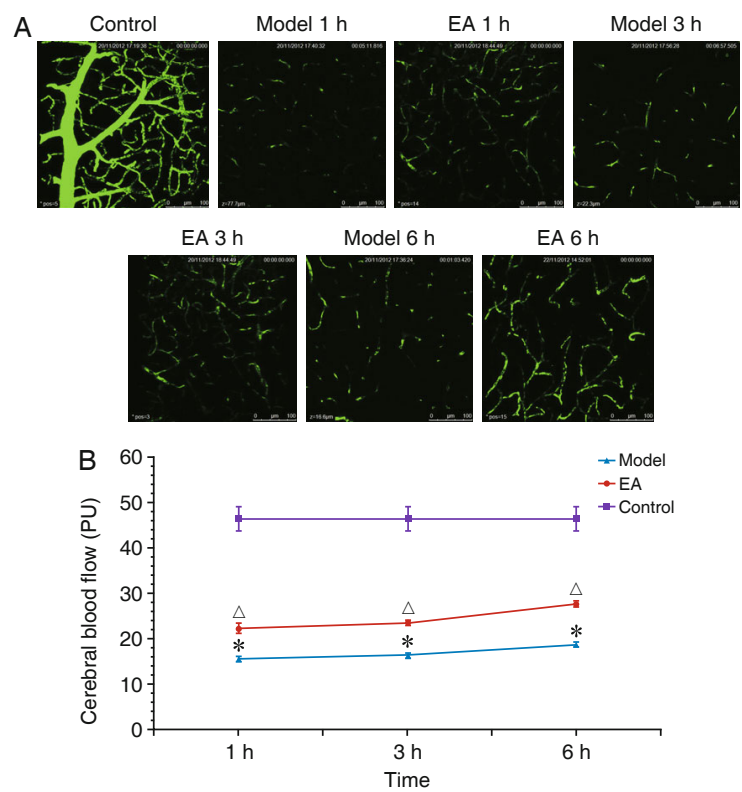

Figure 2. EA Induced Increase of Cerebral Blood Flow in Rats with Acute Cerebral Infarction $(\bar{x} \pm s, n=6)$

Notes: A: results of cerebral blood flow detected by laser scanning confocal microscope; B: comparison of cerebral blood flow among groups. ${ }^{*} P<0.01$ vs. control group at the same time point; ${ }^{\triangle} P<0.01$ vs. model group at the same time point

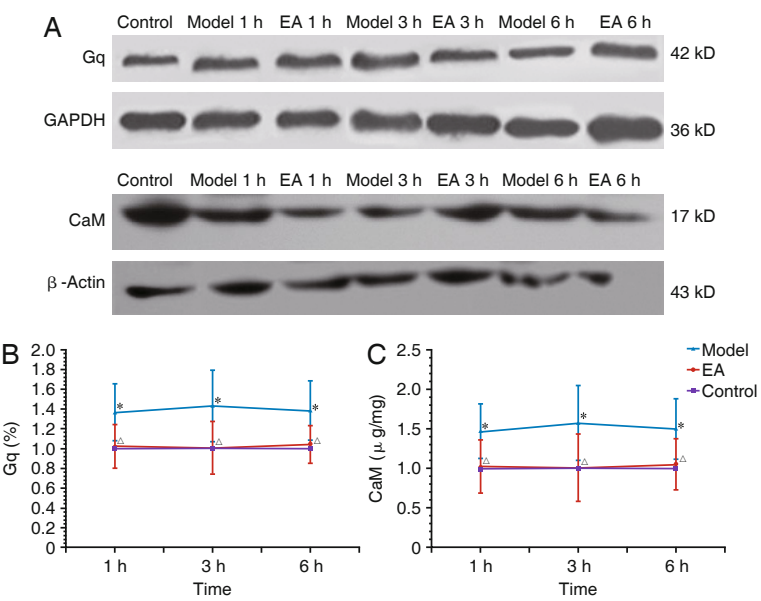

Figure 3. EA Decreased Protein Expressions of $\mathrm{Gq}$ and CaM in Rats with Acute Cerebral Infarction $(\bar{x} \pm s, n=6)$

Notes: ${ }^{*} P<0.05$ vs. control group at the same time point; ${ }^{\triangle} P<0.05$ vs. model group at the same time point

were significantly higher than the control group at the same time points $(P<0.01)$, while the EA group was significantly lower than the model group at the same time points $(P<0.01$, Figure 4$)$.

\section{EA Reduced Contents of $\left[\mathrm{Ca}^{2+}\right]_{i}$ in Rats with} Acute Cerebral Infarction

Compared with the control group, the content of $\left[\mathrm{Ca}^{2+}\right]_{i}$ in the model group rapidly increased and reached a peak within $3 \mathrm{~h}$ after MCAO $(P<0.01)$. The

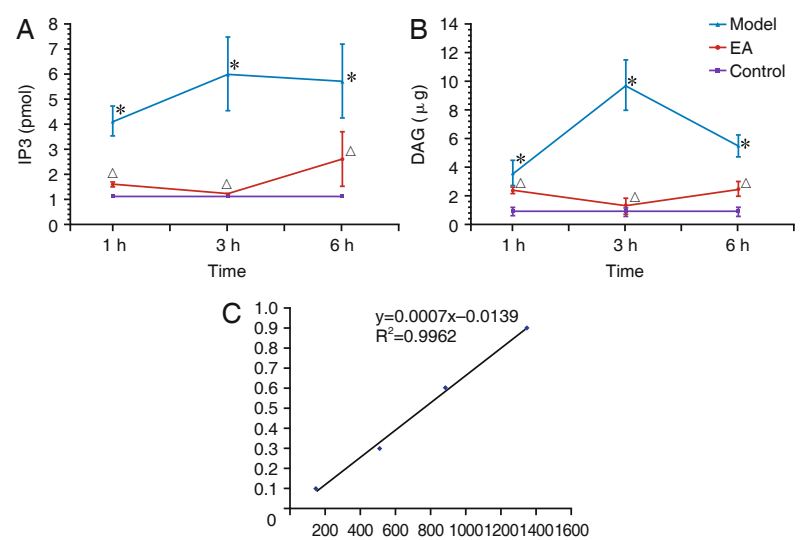

Figure 4. EA Reduced Contents of IP3 and DAG in Rats with Acute Cerebral Infarction $(\bar{x} \pm s, n=6)$

Notes: $A$ and $B$ : contents of IP3 and DAG. ${ }^{*} P<0.01$ vs. control group at the same time point; ${ }^{\triangle} P<0.01$ vs. model group at the same time point. C: DAG standard curve. The regression equation was $y=0.0007 x-0.0139$, and the correlation coefficient $R^{2}$ was 0.9962 . It shows that linear regression is better and can be analyzed quantitatively.

EA group showed a continuous downward trend, in which the $\left[\mathrm{Ca}^{2+}\right]_{i}$ content was significantly lower than the model group at 1 and $3 \mathrm{~h}(P<0.01$, Figure 5$)$.

\section{DISCUSSION}

The present study used the 18-point NSS method to correlate the focal cerebral infarction with $\mathrm{EA}$, and found that EA at GV 26 could improve the symptoms of neurological deficits in MCAO model rats. After the onset of cerebral infarction, the collateral vascular system on the brain surface acts as a "gate", ${ }^{(15)}$ which determines whether the cerebral ischemic area can effectively receive the collateral circulation blood flow from the peripheral ischemic area in time. It also pressurizes the compensated blood flows into the fine blood vessels to nourish ischemic neuronal tissue. When acute cerebral infarction occurs, the resistance of the collateral flow on the surface of the brain increases. The self-regulating movement of microvasculature is visible as high frequency and low amplitude, and the dynamic coupling of fluid and flow field gets obstructed. It is characterized by "high speed and ineffective oscillation", ${ }^{(16)}$ which prevents the brain of the ischemic tissue from getting the compensatory blood flow.

Acupuncture has become an important measure for rehabilitation after stroke. ${ }^{(17)}$ Our previous studies have demonstrated that EA at GV 26 can regulate the effect of cerebral vessels and promote the collateral circulation of cerebral vascular system, which is an important therapy after the onset of cerebral ischemia. ${ }^{(5-7)}$ In the present study we found that the cerebral blood flow in the model 


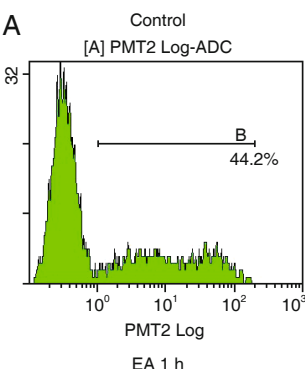

A] PMT2 Log-ADC

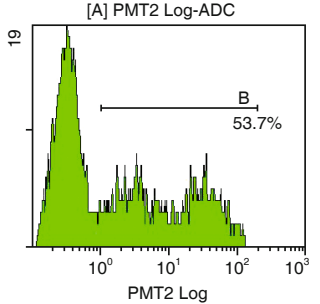

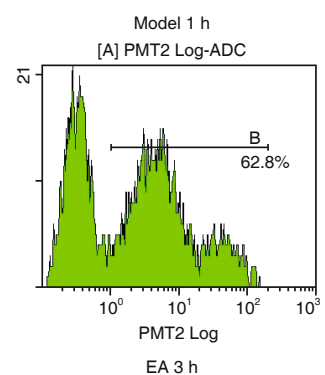

[A] PMT2 Log-ADC
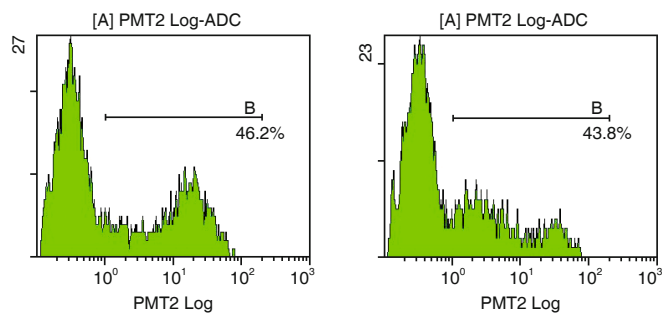

Model $3 \mathrm{~h}$

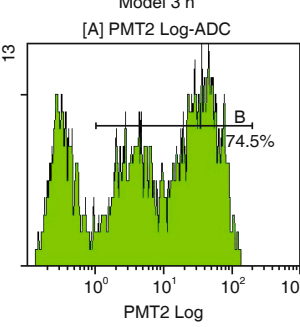

PMT2 Lo

EA $6 \mathrm{~h}$
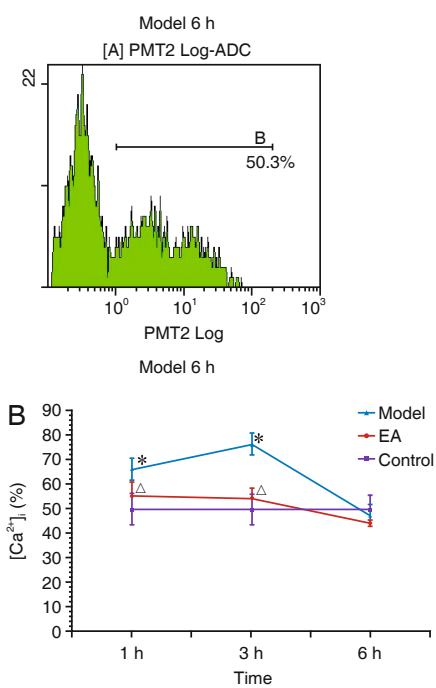

Notes: ${ }^{*} P<0.01$ vs. control group at the same time point; ${ }^{\wedge} P<0.01$ vs. model group at the same time point

group decreased significantly after MCAO compared with the control group $(P<0.01)$, while the cerebral blood flow of EA group was significantly higher than the model group at the same time point $(P<0.01)$. Therefore, we propose that EA could increase blood flow effectively and continuously in the early stage of cerebral infarction (within $6 \mathrm{~h}$ ).

The PI system has been recently found to be closely related to vasoconstriction. ${ }^{(18)}$ The PI system is composed of a molecular switch $\mathrm{Gq}$, an intracellular second messenger IP3, DAG and CaM, which ultimately converts contraction signals into vasoconstriction. As a specific protein that regulates the activity of phospholipase $C$ (PLC)- $\beta 1$, Gq acts as a mediator of membrane receptor and PLC- $\beta$ in inositol phospholipid pathway. ${ }^{(19,20)}$ Changes in its content affect the coupling efficiency of membrane receptors and effectors and in turn affect the ability of cerebral arteries to respond to stimulation. When $\mathrm{Gq}$ is inactivated and $\alpha$ subunit binds to GDP, PLC becomes inactive. ${ }^{(20)}$ When the signal molecule recognizes and binds to the receptor, it leads to a change in the receptor conformation, exposing the site that binds to $\mathrm{Gq}$, and forming a receptor-Gq complex. This leads to a change in the conformation of $\alpha$ subunit of $\mathrm{Gq}$ and it gets separated from the complex of $\beta \gamma$ subunit, repelling GDP, and is thus activated by binding to GTP. ${ }^{(21)}$ The $\alpha$ subunit-GTP complex binds to PLC causing its activation. The activated PLC hydrolyzes phosphatidylinositol 4,5 diphosphate on the plasma membrane to produce IP3 and DAG. With the hydrolysis of GTP, the $\alpha$ subunit restores its original conformation and dissociates from PLC, terminating the activation of PLC. After the reorganization of the $\alpha$ subunit and the $\beta \gamma$ subunit, the cells return to a quiescent state. ${ }^{(22)}$ Our results were in concordance with the above observations as the Gq protein expression in the model group was significantly higher than that of the control group $(P<0.05)$, while the same was significantly lower for the EA group as compared to the model group $(P<0.05)$, suggesting that stimulating GV 26 could effectively decrease the $\mathrm{Gq}$ expression.

IP3 is a cell signal transduction molecule that has attracted increasing attention in recent years. Previous studies revealed that IP3 combines with a specific receptor in the calcium pool, causing activation of $\mathrm{Ca}^{2+}$ dependent pathways, and releasing $\mathrm{Ca}^{2+}$ in the cytoplasm, thereby regulating vasoconstriction. ${ }^{(23,24)}$ Under normal circumstances, DAG only exists as a transient intermediary in the cell membrane for a very short time. In recent years, the study of smooth muscle cells and the pituitary cells showed that activated protein kinase $C$ (PKC) decomposes the phosphatidylinositol 4,5-bisphosphate (PIP2) creating free PI and phosphatidylinositol-4-phosphate (PIP). As a result, the production of DAG exceeds IP3, which may help maintain physiological effect of agonists. DAG and IP3 act as the second messenger to activate different signaling pathways. A combination of DAG mediated activation of DAG/PKC signaling pathways (involved in various physiological activities by the PKC pathway), and IP3 mediated activation of IP3/ $\mathrm{Ca}^{2+}$ signaling pathway, along with specific receptors in endoplasmic reticulum cells causes a release of $\mathrm{Ca}^{2+}$ in calcium library. This increase in the concentration of $\mathrm{Ca}^{2+}$ 
activates the $\mathrm{Ca}^{2+}$ signaling system which regulates various physiological process and the activity of calcium binding protein of enzyme. In this study, the levels of IP3 and DAG in the model group were significantly increased compared with the control group $(P<0.01)$, which is consistent with previous research results. ${ }^{(25)}$ Compared with the model group at the same point, the expressions of IP3 and DAG in the EA group were significantly lower $(P<0.01)$, suggesting that stimulating GV 26 could effectively impact the expression of IP3 and DAG after the onset of cerebral ischemia.

Ischemic stimulation occurs through a series of events acting on the cerebral artery cell, which also causes an increase in the levels of $\mathrm{Ca}^{2+}$. Changes in $\mathrm{Ca}^{2+}$ concentration have been reported to regulate the relaxation function of vascular smooth muscle as the intracellular $\mathrm{Ca}^{2+}$ concentration is an important link in the excitation-contraction of vasculature in smooth muscles. $\mathrm{Ca}^{2+}$ itself has no direct activity, but it acts by combining with $\mathrm{CaM}$ to form a $\mathrm{Ca}^{2+}-\mathrm{CaM}$ complex. ${ }^{(26,27)}$ The $\mathrm{Ca}^{2+}$-CaM complex activates a series of contraction devices causing vasoconstriction. ${ }^{(28)} \mathrm{CaM}$ is the most widely distributed, most functional, and most studied calcium signaling receptor in cells and is closely related to many cell functions such as regulating cell division, cyclic nucleotide metabolism, and DNA synthesis. ${ }^{(29)}$ $\mathrm{CaM}$ is a small soluble globulin molecule consisting of 19 amino acids and is known to be resistant to heat and acid. ${ }^{(30)}$ Due to its strong affinity for $\mathrm{Ca}^{2+}, \mathrm{CaM}$ is the most important calcium receptor in cells. When the intracellular $\mathrm{Ca}^{2+}$ concentration increases, $\mathrm{Ca}^{2+}$ combines with $\mathrm{CaM}$ to form a $\mathrm{Ca}^{2+}$-CaM complex. This complex can activate myosin light chain kinases (MLCK) causing the phosphorylation of the myosin light chain (MLC), leading to contraction caused by myosin activated actin. When the concentration of $\mathrm{Ca}^{2+}$ decreases, CaM separates from MLCK, causing its deactivation and MLCK dephosphorylated, resulting in smooth muscle relaxation. Besides, the $\mathrm{Ca}^{2+}-\mathrm{CaM}$ complex can activate protein kinase, catalyzing the transformation of 1-phosphogluconate to 6-phosphogluconate which in turn gets decomposed, releasing the ATP and providing energy for contraction. The $\mathrm{Ca}^{2+}$-CaM complex can also activate adenylate cyclase $(A C)$, causing an increase in the cAMP concentration, activating the $\mathrm{Na}^{+}-\mathrm{K}^{+} \mathrm{ATP}$ in the cell membrane and sarcoplasmic reticulum, resulting in an increased exchange of $\mathrm{Na}^{+}-\mathrm{Ca}^{2+}$. This increases the intracellular $\mathrm{Ca}^{2+}$ concentration, increasing the $\mathrm{Ca}^{2+}-\mathrm{CaM}$ complex and therefore, leading to enhanced contraction. ${ }^{(31)}$
In the present study, the $\left[\mathrm{Ca}^{2+}\right]_{\mathrm{i}}$ content in the model group was significantly higher than the control group at 1 and $3 \mathrm{~h}(P<0.01)$, indicating that cerebral ischemia could be responsible for this increase. The $\left[\mathrm{Ca}^{2+}\right]_{i}$ content in the EA group at 1 and $3 \mathrm{~h}$ was significantly lower than the model group $(P<0.01)$, indicating that $E A$ could effectively reduce the content of $\left[\mathrm{Ca}^{2+}\right]_{i}$ in the early stage of cerebral infarction ( $3 \mathrm{~h}$ ). The CaM content of the model group was significantly higher than the control group $(P<0.05)$, while the expression of $\mathrm{CaM}$ in the $\mathrm{EA}$ group was significantly lower than the model group at the same point $(P<0.05)$. These results suggest that cerebral ischemia can cause a change of CaM content, and stimulating GV 26 can effectively modulate the expression of CaM after the onset of cerebral ischemia.

Our results also indicate that EA decreased the $\mathrm{Gq}$ expression in signal transduction pathways participating in cerebrovascular contraction. $\mathrm{Gq}$ is a specific binding protein, which can cause cerebral vasoconstriction in PI system pathways. Changes to its content directly affect the IP3 and DAG concentrations in the cell, causing a reduction in intracellular $\mathrm{Ca}^{2+}$ concentration, which was increasing rapidly due to previous ischemic stimulation. At the same time, stimulating GV 26 can decrease the CaM content, reduce the $\mathrm{Ca}^{2+}-\mathrm{CaM}$ complex formed intracellularly, and reduce the vasoconstrictive effect. In a nutshell, EA treatment at GV 26 during the acute phase of cerebral infarction can effectively inhibit the expression of $\mathrm{PI}$ system of the related factors, improve cerebral autonomy movement, alleviate cerebral vascular spasm, and promote vasodilation in early stage. In addition, further studies are required to explore detailed mechanisms of observed effects.

\section{Conflict of Interest}

The authors declare no conflict of interest.

\section{Author Contributions}

$\mathrm{Li} \mathrm{J}$ and Du YH conceived and designed the experiments. $\mathrm{He} Y$ analyzed the data and wrote the paper. Zhang $M$ participated in the production of animal models. Georgi $R$, Kolberg B, Sun DW, Ma K, Li YF and Zhang XZ performed the experiments. All authors read and approved the final manuscript.

\section{Acknowledgement}

Thanks to Lin X (ANANDA Gesundheitszentrum, Berlin, Germany) for her contribution to this article. Lin $X$ participated in the model inclusion criteria. 


\section{REFERENCES}

1. Shin TH, Lee DY, Basith S, Manavalan B, Paik MJ, Rybinnik I, et al. Metabolome changes in cerebral ischemia. Cells 2020;9:1630.

2. Li L, Jiang Q, Zhang L, Ding GL, Zhang ZG, Li QJ, et al. Angiogenesis and improved cerebral blood flow in the ischemic boundary area detected by MRI after administration of sildenafil to rats with embolic stroke. Brain Res 2017;1132:185-192.

3. Li J, He JJ, Du YH, Cui JJ, Ma Y, Zhang XZ. Electroacupuncture improves cerebral blood flow and attenuates moderate ischemic injury via angiotensin II its receptors-mediated mechanism in rats. BMC Complement Altern Med 2014;14:441.

4. Han QD, Wen YY, eds. Vascular biology. Beijing: Beijing Medical University \& China Union Medical University Press;1997:74-79.

5. Du YH, Shi L, Li J, Xiong J, Li B, Fan XN. Angiogenesis and improved cerebral blood flow in the ischemic boundary area were detected after electroacupuncture treatment to rats with ischemic stroke. Neurol Res 2011;1:101-107.

6. Lu Y, Du YH, Xu YL, Gao L, Cui JJ, Yang LH, et al. Study on expression of PKC in vascular smooth muscle of rats with middle cerebral artery occlusion and interfering effect of electroacupuncture at Shuigou. China J Tradit Chin Med Pharm (Chin) 2013;28:2310-2314.

7. Lu Y, Du YH, Xu YL, Gao L, Cui JJ, Yang LH, et al. Effect of electro-acupuncture at "Shuigou" (GV 26) on immunoactivity and content of protein kinase $C$ in the middle cerebral artery in acute cerebral infarction rats. Acupunct Res (Chin) 2015;40:219-223.

8. Longa EZ, Weinstein PR, Carlson S, Cummins R. Reversible middle cerebral artery occlusion without craniectomy in rats. Stroke 1989;1:84-91.

9. Hua XB, Zhou HL. Development of acupoint atlas of rats. Lab Animals Animal Exp (Chin) 1991;1:1-5.

10. Chen J, Li Y, Wang L, Zhang Z, Lu D, Lu M, et al. Therapeutic benefit of intravenous administration of bone marrow stromal cells after cerebral ischemia in rats. Stroke 2001;32:1005-1011.

11. Najm FJ, Madhavan M, Zaremba A, Shick E, Karl RT, Factor DC, et al. Drug-based modulation of endogenous stem cells promotes functional remyelination in vivo. Nature 2015;522:216-220.

12. Wang QL, Xie J, Liang J, Dong GT, Qing LS. Competitive protein binding assay of naproxen by human serum albumin functionalized silicon dioxide nanoparticles. Molecules 2019;24:2593.

13. Zhao Y, Wang C, Qiu B, Li C, Wang H, Jin H, et al. Passive immunotherapy for Middle East respiratory syndrome coronavirus infection with equine immunoglobulin or immunoglobulin fragments in a mouse model. Antiviral Res 2017;137:125-130.

14. Deng YJ, Yi SX, Lin YP, Yan J, Guo H, Xiang ZY, et al. Effects of acupuncture at acupoints of the Foot Yangming meridian on calcium ion concentration of smooth muscle cells in gastric autrum in the rabbit. J Tradit Chin Med (Chin) 2004;15:492-494.

15. Lin X, Du YH. Effect of electroacupuncture on cAMP of cerebral vascular artery smooth muscle cells in rats with acute cerebral infarction. Chin J Basic Med Tradit Chin Med (Chin) 2007;13:860-862.

16. Guan L, Du YH. Effect of acupuncture on automatic rhythmicity in microvasculature of cerebral pia mater in rats with local infarction. Tianjin J Tradit Chin Med (Chin) 2006;3:209-210.

17. Xiong W, Zhao CM, An LX, Xie SN, Jia CR. Efficacy of acupuncture combined with local anesthesia in ischemic stroke patients with carotid artery stenting: a prospective randomized trial. Chin J Integr Med 2020;26:609-616.

18. Jover-Mengual T, Castelló-Ruiz M, Burguete MC, Jorques M, López-Morales MA, Aliena-Valero A, et al. Molecular mechanisms mediating the neuroprotective role of the selective estrogen receptor modulator, bazedoxifene, in acute ischemic stroke: a comparative study with $17 \beta$-estradiol. J Steroid Biochem Mol Biol 2017;171:296-304.

19. Oldham WM, Hamm HE. Heterotrimeric G protein activation by G-protein-coupled receptors. Nat Rev Mol Cell Biol 2008;9:60-71.

20. Qian J, Gu YY, Wu C, Yu F, Chen YQ, Zhu JM, et al. Agonistinduced activation of human FFA1 receptor signals to extracellular signal-regulated kinase 1 and 2 through Gq- and Gi-coupled signaling cascades. Cell Mol Biol Lett 2017;22:13.

21. Patt J, Alenfelder J, Pfeil EM, Voss JH, Merten N, Eryilmaz F, et al. An experimental strategy to probe Gq contribution to signal transduction in living cells. J Biol Chem 2021;296:100472.

22. Kankanamge D, Ubeysinghe S, Tennakoon M, Pantula PD, Mitra $\mathrm{K}$, Giri L, et al. Dissociation of the $\mathrm{G}$ protein $\beta \gamma$ from the $\mathrm{Gq}$ PLC $\beta$ complex partially attenuates PIP2 hydrolysis. J Biol Chem 2021;296:100702.

23. Nassar NN, Abdel-Rahman AA. Brain stem adenosine receptors modulate centrally mediated hypotensive responses in conscious rats: a review. J Adv Res 2015;3:331-340.

24. Litosch I. Regulating G protein activity by lipase-independent functions of phospholipase C. Life Sci 2015;137:116-124.

25. Sun DW, Du YH, Shi L, Liu WH. Effect of electroacupuncture on inositol triphosphate and diacylglycerol levels in cerebral arteries of cerebral ischemia rats. Acupunct Res (Chin) 2008;33:392-396.

26. Singer HA. $\mathrm{Ca}^{2+} / c a l m o d u l i n-d e p e n d e n t$ protein kinase II function in vascular remodelling. J Physiol 2012;590:1349-1356.

27. Saddouk FZ, Ginnan R, Singer HA. $\mathrm{Ca}^{2+} /$ calmodulin-dependent protein kinase II in vascular smooth muscle. Adv Pharmacol 2017;78:171-202

28. Saddouk FZ, Sun LY, Liu YF, Jiang M, Singer DV, Backs J, et al. $\mathrm{Ca}^{2+} /$ calmodulin-dependent protein kinase II - $\gamma$ (CaMK II $\gamma$ ) negatively regulates vascular smooth muscle cell proliferation and vascular remodeling. FASEB J 2016;30:1051-1064.

29. Tebar F, Chavero A, Agell N, Lu A, Rentero C, Enrich C, et al. Pleiotropic roles of calmodulin in the regulation of KRas and Rac1 GTPases: functional diversity in health and disease. Int $J$ Mol Sci 2020;21:3680.

30. Aseervatham J, Li X, Mitchell CK, Lin YP, Heidelberger R, O'Brien J. Calmodulin binding to connexin 35: specializations to function as an electrical synapse. Int J Mol Sci 2020;21:6346.

31. Tzortzopoulos A, Best SL, Kalamida D, Török K. $\mathrm{Ca}^{2+}$ / calmodulin-dependent activation and inactivation mechanisms of alphaCaMK II and phospho-Thr286-alphaCaMK II . Biochemistry 2004;43:6270-6280

(Accepted January 8, 2021; First Online December 7, 2021) Edited by YU Ming-zhu 